
\title{
28 Research Suare \\ Passive exoskeleton assisted treadmill walking reduces duration and synchrony of inter-limb coordination
}

\section{Takashi Sado}

University of Nebraska at Omaha

\section{Zachary Motz}

University of Nebraska at Omaha

Jennifer M. Yentes

Department of Health \& Kinesiology, Texas A\&M University

Mukul Mukherjee ( $\nabla$ mmukherjee@unomaha.edu )

University of Nebraska at Omaha

\section{Research Article}

Keywords:

Posted Date: February 23rd, 2022

DOI: https://doi.org/10.21203/rs.3.rs-1327967/v1

License: (c) (i) This work is licensed under a Creative Commons Attribution 4.0 International License.

Read Full License

Version of Record: A version of this preprint was published at Frontiers in Physiology on June 13th, 2022. See the published version at https://doi.org/10.3389/fphys.2022.916185. 


\section{Abstract}

Exoskeleton assistive devices have been developed as a potential approach to solve gait deficits like paretic propulsion and reduced speed. However, it is unclear how these devices affect inter-limb coordination. The duration and the synchrony of gait coordination was assessed during passive exoskeleton-assisted walking in healthy young individuals. It was hypothesized that inter-limb coordination would be reduced in comparison to normal walking without assistance, thus demonstrating gait with exoskeletal to be more explorative and flexible. All participants performed 5 minutes walking at a preferred walking speed after a familiarization trial. The duration of inter-limb coordination was examined using cross-recurrence quantification analysis (CRQA) and the synchrony between the interlimb coordination was measured using cross sample entropy (CSE). There were no significant differences in spatiotemporal measurements between the two groups. However, in comparison to the NO EXO group, there was a reduction in the duration of coordination (mean diagonal length: $p<0.01$ ) and the synchrony of coordination (entropy value: $\mathrm{P}<0.05$ ) in the EXO group. These results indicate that exoskeletal-assisted gait is characterized by reduced inter-limb coordination possibly for allowing gait patterns to be more explorative and flexible. This is important in rehabilitation of patients who suffer from coordination deficits.

\section{Introduction}

Inter-limb coordination is a term that characterizes the movement of one limb in relation to another during specific tasks such as walking. In a healthy human, this coordination is smooth and shows flexibility to different tasks and environments. Inter-limb coordination is affected by the neural linkage between limbs and is flexible in a way that can be adjusted to different task-environments ${ }^{1}$. Two legs can be coordinated perfectly as in a robotic walking system ${ }^{2}$, or not coordinated, resulting from random movements as in ataxic gait. Neither of these demonstrate optimal coordination, a hallmark of healthy natural gait. This is because when stressful environments or tasks are encountered (e.g. sideways perturbation), both of these coordination patterns generally fail, leading to falls.

Based on the above, a theoretical model of coordination can be considered as shown in Fig. 1. In this model, we see two extremes of coordinative states that exhibit either metronomic qualities or random-like qualities. Both these states can be indicative of unhealthy pathological disease processes. Healthy interlimb coordination sits at a sweet-spot between these systems. Healthy natural gait is characterized by two limbs effectively working together providing smooth, flexible, and efficient gait ${ }^{3,4}$. In disease processes like stroke or Parkinson's disease, this optimal coordination is affected ${ }^{5,6}$.

For example, hemiparetic disease, like stroke can cause people to display larger between leg phase-shifts in response to a perturbation compared to healthy controls ${ }^{7}$. Inter-limb phasing using cross-correlation showed characteristic adaptive changes in bilateral coordination tasks like the split-belt adaptation task in stroke survivors ${ }^{8,9}$. In other neurological conditions, like Parkinson's disease, people showed reduced inter-limb coordination compared to healthy controls ${ }^{6,10,11}$. A traditional measure, phase coordination 
index, was significantly increased in people with Parkinson's disease compared to a healthy sample, indicating worse bilateral coordination ${ }^{10,11}$. Another coordination variable, cross-covariance was utilized to show that ipsilateral and contralateral inter-limb coordination was reduced in people with Parkinson's disease ${ }^{6}$. For such patient populations, who have been affected by neurological diseases, an exoskeletal system such as a wearable robotic gait-assistive device, may have potential to improve inter-limb coordination.

For many years, researchers have shown how active (powered) and passive exoskeletons (unpowered) affect how people perform different walking tasks ${ }^{12-15}$. In the field of exoskeleton research, most of the effort has been directed towards the reduction of energy expenditure and muscle activations, and these revealed the mechanical benefits of the exoskeleton assistance $2,14,16$. However, it is still not known how such assistive devices change inter-limb coordination during walking. In this study, a passive exoskeleton was used. This device ${ }^{17}$ was originally built for patients like stroke survivors or spinal cord injury. In brief, this device has a band that runs through ankle, knee, and hip, and is attached to a spring. During the stance phase of walking, the band pulls the spring, stores energy, and returns the energy as the toe propels forward to help swing the leg forward ${ }^{17,18}$.

Exoskeleton assistance can potentially alter inter-limb coordination by making gait either a) more periodic, i.e., like a sinusoidal wave or b) more random. It is also possible that exoskeleton-assisted gait may show no change in coordination in comparison to walking without such assistance. While healthy young individuals can produce somewhat periodic, but complex fluctuations ${ }^{19}$, it is not well understood how exoskeleton-assistance affects inter-limb coordination. Therefore, the purpose of this study is to determine which specific direction exoskeletal-assisted gait would take inter-limb coordination patterns toward - more periodic or more random as in Fig. 1. The clinical implication of such an alteration would inform the development of devices that restore healthy inter-limb coordination in patients.

To quantify coordination, traditional coordination measurements include discrete relative phase ${ }^{20}$, continuous relative phase ${ }^{21}$, cross-correlation ${ }^{22}$, and normalized root mean squared difference ${ }^{23}$. However, each of these methods have certain limitations which prevent a complete picture of the complex dynamic nature of gait coordination from emerging. These measurements provide discrete outcomes based on the time series which only look at snapshots of movements. However, human movements are rather dynamic and continuous and are like movies. These linear measurements may be sufficient to describe some parts of dynamical systems, but it may not tell the whole story. This is of critical importance in special populations, like stroke and unilateral amputees, where one half of the body was impaired by the disease with characteristic effects distinctly different from the other half. Therefore, in these situations, the overall effect is one of an abnormally coordinated multi-segment unit. In addition, both discrete relative phase and continuous relative phase are based on the assumptions that the two limbs during gait, are at a one-to-one frequency, or completely periodic like a sine wave ${ }^{24}$. Unless appropriate variables are used to assess such abnormal behavior, the impact on therapy and rehabilitation may not be significant. Examples of such assessment instruments are cross recurrence 
quantification analysis (CRQA) and cross sample entropy (CSE). These nonlinear methods of analyses can capture the evolution of the movements and quantify the coordination between the left-and rightlegs.

The CRQA technique is a modified version of recurrence quantification analysis (RQA) and quantifies how long two different dynamical systems exhibit coordinated patterns of behavior over time ${ }^{25}$. In this analytical method a recurrence plot is created as a result of reducing a higher-dimensional, nonlinear pattern into two-dimensions, which shows the recurring patterns or trajectories of that dynamical system $^{26}$. Changes in physiological or environmental dynamics alter the duration of coordination ${ }^{26,27}$, in effect quantifying the three aspects of our theoretical model in Fig. 1. Sample entropy is a nonlinear analysis method which quantifies the regularity or predictability of a single time series ${ }^{28}$. Larger sample entropy values indicate more irregularity in a time series. While sample entropy examines the regularity of a single time series, CSE measures the synchrony between two time series. In other words, it examines whether there are similar patterns between two data series ${ }^{26}$ with larger cSE values indicating more asynchrony.

The focus in our study was to determine how inter-limb coordination was affected through exoskeletonassisted gait. It was hypothesized that imposed passive exoskeleton assistance would lead to a change in the dynamics of inter-limb coordination. This will be demonstrated with a reduction in duration of coordination and synchrony of gait patterns without changing conventional gait measurements like step length, step time, step width, and walking speed. Specifically, we hypothesized that inter-limb coordination would show characteristic decreases in coordination duration and synchrony when walking at preferred walking speed (PWS) with a unilateral exoskeleton device if the device helped to make gait more explorative. Alternatively, these two measures would be flipped, i.e., increased duration and synchrony, if exoskeletal-assisted gait resulted in increased restriction.

\section{Results}

\section{Subject Demographic}

Participant demographics are shown in Table 1. There were no significant differences between the groups for anthropometric measures. The two groups did not differ in their PWS. The mean and standard deviation of spatial and temporal measures showed no significant differences between the two groups during treadmill walking at their PWS (Table 3). Mann-Whitney $U$ test showed no difference between the groups for EMB and step length.

Analysis of inter-limb coordination patterns through CRQA and CSE showed significant group differences. State-space reconstruction figures for one participant performing the treadmill walking task without the exoskeleton (A) and another participant performing the task with the exoskeleton (Figure 2B) shows the characteristic evolution of each heel marker trajectory over time. Recurrence plots are presented for one 
participant performing the treadmill walking without the exoskeleton (Figure $3 \mathrm{~A}$ ) and another participant performing the task with the exoskeleton (Figure 3B).

\section{Cross Recurrence Quantification Analysis Result}

Significant group differences in the CRQA parameters (\%DET, Radius, and MeanL) are shown in Figure 4. Specifically, there was no difference in EMB. Meaning the dimensionality (information) required to provide an accurate assessment of coordination behavior is not different between the groups. Radius was significantly larger $(t(16)=3.741, p=0.002)$ in the EXO group $(120.64 \pm 22.91$ points) compared to the NO EXO group (88.49 \pm 11.82 points). This means that, for EXO group, a larger radius was required to incorporate the same number of recurrent points as the NO EXO group. This may be an indication of reduced interactions between the limbs. Percent determinism (\%DET) did not show significant difference between groups. No difference in \%DET suggests that wearing an exoskeleton does not change the determinism of coordination between the two limbs compared to the NO EXO group. Mean diagonal length (Mean $\mathrm{L}$ ) was significantly shorter $(\mathrm{t}(16)=-3.228, \mathrm{p}=0.005)$ in the EXO group $(7.65 \pm 1.33$ points) compared to the NO EXO group ( $9.31 \pm 0.80$ points), which indicates that average duration time that the two limbs were coordinated was shorter in EXO group.

\section{Cross Sample Entropy Results}

CSE was shown to have a significantly higher value $(t(16)=2.552, p=0.021)$ in EXO group $(0.20 \pm 0.026$ bits) compared to NO EXO group ( $0.16 \pm 0.03$ bits), indicating increased asynchrony of heel trajectories between the right and left feet when walking with the exoskeletal device compared to natural gait.

\section{Discussion}

The purpose of the current study was to examine how passive assistance, provided by an exoskeletal device would lead to a change in the dynamics of inter-limb coordination, which could be demonstrated with a reduction in duration of coordination and synchrony of gait. Our results provided evidence in favor of both primary hypotheses-walking with the device showed inter-limb coordination patterns that were of shorter duration and lower regularity than walking without the device. This indicated that assistance from the exoskeleton had potential to make gait more exploratory rather than more restricted. Further, this was achieved without any associated differences in the linear measures (Table 2).

No differences were found in average step length, width, and time between the two groups. The participants walking at their PWS, adapted to the exoskeleton without requiring spatio-temporal adjustments. Walking with a unilateral passive spring-loaded exoskeleton was not a complicated task. Additional exoskeleton studies in healthy ${ }^{13,29}$ and pathological populations ${ }^{12,17}$ do not show changes in gait patterns during treadmill walking. Although the design of exoskeletons with unilateral or bilateral placement may have effects on step width, typically it has been found that step width is less likely to be affected by the exoskeleton ${ }^{30,31}$. This may also be due to the requirement of staying at the center of the treadmill (both anteroposterior and mediolaterally) at constant speed 
In our work with stroke survivors, we found that walking with this device for five days changed spatiotemporal patterns, specifically step time, length and width and double support time ${ }^{18}$. The differences with results here could be due to healthy participants in this study who would be generally more adaptive, had less exposure to the EXO device, and were walking on a treadmill instead of over ground.

Determination of the EMB is an important step towards reconstructing the time series to maximize the information that can be extracted from a complex nonlinear system. This can be further visualized through state space reconstruction (Fig. 4). Walking with the device did not change how many dimensions were needed to maximize the information extracted from the dynamical system. In healthy young individuals, state-space reconstruction of normal treadmill walking showed a tear-drop shape (Fig. 4A), and this structure was maintained even when people walked with the exoskeleton (Fig. 4B).

The radius, however, was larger in the EXO group-to assess the coordination duration between the limbs, a larger radius was need while the recurrence rate was kept to $2.5 \%$. This means that two trajectories were further apart for the EXO group. It can be speculated that human coordination has certain constraints that makes it healthy. A passive exoskeleton assistance may remove these constraints, making people to be more explorative. However, this does not mean that smaller radius causes the duration of coordination to be shorter.

There were no differences in the \%DET between the EXO and the NO EXO group. If coordination patterns were highly deterministic as in robotic gait, \%DET would have been close to $100 \%$. In this study, we found that \%DET was approximately $90 \%$ in both groups, demonstrating that inter-limb coordination in healthy young participants was characterized by a high degree of determinism. Specifically, the chances of finding recurrent points forming diagonal lines in the recurrence plot was high, around $90 \%$. A similar level of determinism has been shown in healthy humans when coordination between different physiological systems such as breathing and walking were considered ${ }^{26}$. When the sensorimotor systems are affected, such as in Parkinson's disease ${ }^{32}$ and hypovestibular disorders ${ }^{33}$, recurrence patterns were demonstrated to be less deterministic in comparison to healthy. In postural tasks, \%DET has been shown to be consistently high in healthy participants and were affected by specific tasks such as maintaining balance during high frequency oscillations of the support surface ${ }^{27}$. In that study, \%DET was reduced significantly only when oscillations crossed a specific threshold and became too difficult. In our study, it was shown that addition of a unilateral exoskeletal device did not affect the determinism of recurrence points on the diagonal lines. Therefore, our task of walking at PWS being a simple task and the participants being healthy essentially led to both groups being similarly deterministic.

Walking with the exoskeleton led to a reduced average time that the two limbs were coordinated at PWS compared to those that did not wear the exoskeleton. Theoretically, robotic gait is likely to produce very long mean diagonal lengths because each limb produces periodic sinusoidal movements that repeat perfectly. Contrarily, there is an inherent variability in healthy human dynamical systems ${ }^{34}$ and such variability is characterized in the recurrence plots $(A)$. When healthy young individuals walked with their 
limb loaded unilaterally, their inter- and intra-limb coordination (continuous relative phase and cross correlation) were affected such that inter-limb coordination measures reduced ${ }^{24}$. In our theoretical model, duration of coordination was reduced when walking with the device. Specifically, in Fig. 1, exoskeletal assisted gait guided the system towards the right side the model. This was possibly due to reduced coordination between the limbs allowing for greater exploration, instead of tighter coordination that potentially restricted adaptive flexibility. Alternatively, it is possible that the weight of exoskeleton influenced reduced coordination and teasing out the effect of the device weight would require further investigations.

In pathology such as $\mathrm{COPD}^{26}$, coupling between physiological time series such as breathing and walking became more rigid with longer mean lengths in comparison to healthy participants. This was believed to indicate the reduced coupling between physiological systems in healthy participants allowed for greater variability and essentially stemmed from a need to be more flexible and adaptable. Intuitively, that the changes induced with the passive exoskeleton maybe an indication of more flexible and adaptive behavior that such an artificial system allows. This may provide a window of opportunity to make unhealthy, restrictive gait such as in stroke, more adaptive and flexible.

Further, the exoskeleton-assistance reduced the synchrony between the two limbs during walking at PWS. Jordan et al. (2006) showed that when people walk at PWS, they have the lowest a-value (using detrended fluctuation analysis) in comparison to non-preferred speeds (higher/lower than PWS) where strength of long-range correlations increase ${ }^{35}$. This has been understood to be the result of an increase in dynamical constraints and therefore, a reduction in the available degrees of freedom as we move away from PWS. In our study, it appeared that adding a spring-loaded, unilateral, passive device enabled the participants to increase these degrees of freedom and walk with a greater explorative ability. Similar to the addition of an exoskeleton, adding sensory stimuli such as vibratory insole tactors ${ }^{36}$ or auditory feedback ${ }^{37,38}$ changed dynamical patterns during walking. Taking these results further, we have shown that adding assistive constraints led to changes in inter-limb coordination dynamics specifically to the synchrony of these coordination patterns such that walking became less repeatable and more adaptive.

Our finding indicated that a passive exoskeleton induced more exploration by changing the coordination between the limbs. Such changes could be very important for those patients whose movements are constricted. A passive exoskeleton reducing the recurrent gait patterns (less duration and synchrony) can be an indication of error-based learning 39,40 . That is, there is a potential that the exoskeleton assistance may remove coordination constraints, allowing you to explore the task and environment more, so the person can learn something new or different by making errors. In many pathological cases such as neurological deficits or sensorimotor deficits, abnormal coordination was shown $4,11,41$. A passive exoskeleton has the potential to break such restrictive abnormal coordination patterns, allowing patients to learn healthy coordination, helping patients restore their healthy inter-limb coordination. Future work could assess such coordination dynamics during inter-limb coordination tasks such as split-belt adaptation. 
In this study, some limitations were identified. One of the limitations was a between-subjects design. A within-subjects approach may provide more information about change in intra-person behaviors. Although it appears everyone adapted to the device, there were no measurements of adaptation. Withinsubjects test may also show how a person adapts to an assistive device. Also, treadmill walking is known to alter gait compared to overground walking ${ }^{42}$. Examining inter-limb coordination on overground walking using nonlinear tools may reveal different effect of the exoskeleton assistance.

For the exoskeleton that was used for this study, special shoes had to be worn, which may have affected the way people walk. However, participants were given five minutes familiarization trial to get adjusted to walking in this device. Additionally, there was no objective way to know the tightness of the tendon. In a related study ${ }^{18}$, the number of ratchet clicks on the disc at the waist was used to have a generic measure of the exotendon tightness. The EXO group only had an exoskeleton on the one side. It is still unknown whether the reduced duration and synchrony of inter-limb coordination was due to the weight of the device or asymmetry between the limbs. To answer these questions, our future direction should focus on how unilateral and bilateral limb loading changes the specific coordination measurements.

The current investigation focused on how a passive exoskeleton assistance affected the duration and the synchrony of inter-limb gait coordination. It was found that walking with passive assistance decreased the duration and the synchrony of coordination between the limbs. This could indicate that patient populations with abnormal inter-limb coordination could utilize such assistive devices, the assistance could disrupt the abnormal coordination between the limbs, allowing the wearers to explore the environment, and possibly help them to restore healthy coordination.

\section{Methodology}

Participants: In this study, 18 young healthy individuals (19 to 35 years) were recruited and agreed to participate. Recruited participants did not have any lower limb dysfunction, cognitive or neurological impairments, nor vascular or other abnormalities that could affect walking on a treadmill. All participants had previous exposure to treadmill walking. No participants were excluded. Prior to the experiment, each participant signed an informed consent approved by the Institutional Review Board from University of Nebraska Medical Center. All procedures were approved by, and conducted according to the guidelines of, the University of Nebraska Medical Center Institutional Review Board.

Research design: Participants were randomly placed into either a group that walked with the passive exoskeletal device (EXO) or a group that walked without the exoskeleton device (NO EXO). To select their PWS, all participants walked at a self-selected comfortable speed, similar to normal, everyday walking. Speed was increased gradually as the participants walked on the treadmill and participants were instructed to let the examiner know when the PWS speed was reached. All participants first walked on the treadmill for a familiarization trial for five minutes. Then, for those participants who were placed in the EXO group, participants had the device attached to their right dominant leg. The dominance of the leg was determined by asking participant which leg they would use to kick a ball. After the exoskeleton was 
attached, participants confirmed if their PWS was comfortable or it needed to be changed. None of the participants requested to change their speed. Participants in the EXO group had an extra familiarization trial where they walked on the treadmill for five minutes while wearing a passive exoskeleton.

To collect experimental data, all participants were asked to walk for five minutes at their PWS.

Movements of the participants were captured using a digital motion capture system at $100 \mathrm{~Hz}$. (8-camera T160, Vicon, Oxford, UK). Participants wore tight-fitting suits to avoid capturing the movement of clothing. Each participant had 11 markers attached to specific anatomical landmarks that included the anterior superior iliac spine, posterior superior iliac spine, sacrum, lateral malleolus, head of the second metatarsal, and the calcaneus ${ }^{43}$.

Exoskeleton: The exoskeleton device, used in this project, was the Kickstart ${ }^{\mathrm{TM}}$ Walking System (Cadence Biomedical, Seattle, WA). This passive device (Figure 2) used an elastic band (Exotendon ${ }^{\mathrm{TM}}{ }^{17}{ }^{17}$ inspired by a biomechanical model ${ }^{44}$. The band ran parallel to the lateral side of the leg and through pulleys over the three joints (ankle, knee, and hip). The knee joint of the exoskeleton was aligned to the participants' medial-lateral rotational axis at their knee. The exoskeleton was fitted with special shoes, similar to a boot one would wear after breaking a toe (Figure 5). The exoskeletal device was adjusted to each participant at their waist, thigh, and shank. The device was firmly fitted without causing any physical discomfort to the participants. Tightness of the band was adjusted using a disk located on the side of the waist belt. The band was first tightened to the point where all slack was taken up, then it was tightened until participants felt the assist. This was confirmed by having participants walk overground with the device for a minute.

Data analysis: All data processing was done with custom code (Matlab 2019b, Mathworks, Natick, MA). Step length $(\mathrm{m})$, step time (sec), and step width $(\mathrm{m})$ were measured as basic outcomes of performance. Step length was defined as the distance from the heel-strike to contralateral heel-strike in the anteriorposterior direction. Treadmill belt movement was added to calculate step length. Step time was calculated for each side as the duration of time between the contralateral heel strike to the ipsilateral heel strike. Step width was defined as the mediolateral distance between heel markers at successive heel strikes. For each leg, right and left, the first 200 steps were averaged for each of the spatiotemporal outcomes.

The raw marker data from the left and right heel (Figure 6) sagittal plane (anterior-posterior direction) were used for analysis of CRQA and CSE (The custom code is available in the supplemental section of McCamley et al 2017, doi: 10.1155/2017/7960467)26. Specifically, 12,000 data points were unfiltered and down sampled by $10 \mathrm{~Hz}$ to 1,200 data points for further analysis.

As a part of $C R Q A$, state-space was reconstructed for each right and left pair of heel marker data. Time delay $\left(\tau\right.$, tau) was calculated via average mutual information method ${ }^{45}$ for each leg. The first minimum tau value was used. Optimal embedding dimension was computed with the false nearest neighbor analysis ${ }^{46}$. Embedding dimension for each participant and leg were calculated separately (Table 3), and 
the maximum embedding dimension was recorded. When the embedding dimension of the two legs was different, the higher value was used.

Using the computed $\tau$ and embedding dimension, right and left heel markers were reconstructed into their state space. To determine if the time series were recurrent in the same state space, a tolerance window (i.e. radius) was used. The size of the radius fluctuated between participants to ensure a $2.5 \%$ recurrence rate. The recurrence rate was selected based on the assumption that sparse recurrence plots with scattered points reveal the most information ${ }^{47-49}$. The resultant output was a two-dimensional recurrence plot. Each point on the recurrence plot represents a point in time the movement between two legs are coordinated. Once the recurrence plot was constructed, the length of diagonal lines, defined as a minimum of two recurrent points in length, were quantified. The following CRQA features of the recurrence plot were extracted to inform different dynamics of the system.

i. Embedding Dimension (EMB): A time-series needs to be unfolded to the maximum embedding dimension ${ }^{48}$ to ensure that all of the meaningful dynamics are observed. No new information will be found if the movement goes above the determined EMB. If EMB is similar between groups, the dimensionality required to provide an accurate assessment of coordination behavior is not different between the groups. Biologically speaking, it would mean that both groups demonstrate optimal interlimb relationships at similar dimensionality with no new information added if dimensionality is increased.

ii. Radius: the tolerance or cut-off value for the distance between the trajectory of one system (e.g. left leg) and another system (e.g. right leg) to be counted as recurrent. To maintain the recurrence rate of $2.5 \%$, radius fluctuated from participant to participant. A larger radius indicated the limb trajectories were further apart (increased interaction between the limbs) and when smaller, the trajectories were closer together (reduced interaction).

iii. Percent determinism (\%DET): was the percentage of recurrent points falling on a diagonal line compared to all points on the plot. This value is interpreted as the determinism of how two signals are coordinated. A \%DET closer to $100 \%$ indicated an almost perfectly coordinated system.

iv. Mean Diagonal Line Length (MeanL): was the average length of the diagonal recurrence lines and indicated the average time that the two signals spent in the same region of the reconstructed phase space.

Cross sample entropy (CSE) was the probability that patterns in one time series will appear in the other time series. CSE used input parameters of the length of data, vector length, and tolerance. Specific calculation of the CSE can be found elsewhere ${ }^{50}$. In brief, it divided the two different time series with the same length into short vectors of length $m$. For each of these vectors, it compared a vector from one time series to a vector from the other time series to determine whether the vectors are similar. Two vectors were considered similar if they were within the tolerance, $r$. This process was repeated with vectors one longer (i.e. $m+1$ ). Based on the recommendations from ${ }^{26}$, vector length, $m$, was set to 3 . Tolerance, the allowable region of data to be considered a match in patterns, was set to 0.25 * standard deviation. 
In total, the dependent variables subjected to analysis were step length, step time, and step width; cRQA $\mathrm{EMB}$, radius, \%DET, MeanL; and CSE. All dependent variables were examined for normality using a Shapiro-Wilk test. EMB failed normality check. Step length R for EXO and step length L for NO EXO failed the test of normality distribution as well. Transformation of data did not resolve the normality violation. Therefore, we performed Mann-Whitney U nonparametric tests for EMB and step length (left and right). Statistical differences for rest of the measurements (\%DET, MeanL, radius, and CSE) were examined via independent t-tests between EXO and NO EXO. For all statistical tests, alpha level was set at 0.05 . Statistical analysis was done using SPSS (version 26, IBM Inc., Armonk, NY).

\section{Declarations}

\section{Data availability}

The datasets generated during and/or analyzed during the current study are available from the corresponding author on reasonable request.

\section{Acknowledgments}

This study was supported by the Center of Biomedical Research Excellence grant from NIGMS/NIH, a NASA EPSCoR Research grant, and an AHA AIREA award. In addition, intramural grants from the University of Nebraska at Omaha - The Graduate Research and creative activity (GRACA) award and the fund for undergraduate scholarly experiences (FUSE).

\section{Author contributions}

T.S. and M.M. designed the study. T.S. has collected, processed, analyzed the data. T.S., M.M., has prepared all figures and tables. T.S., Z.M., J.Y., and M.M., wrote the manuscript.

\section{Additional information}

Correspondence and requests for materials should be addressed to M.M.

Reprints and permissions information is available at www.nature.com/reprints.

Publisher's note Springer Nature remains neutral with regard to jurisdictional claims in published maps and institutional affiliations.

\section{Ethics declarations}

Competing information: The authors declare no competing interests. All the figures were created and compiled by author T.S.

\section{References}


1. Dietz, V. \& Schrafl-Altermatt, M. Control of functional movements in healthy and post-stroke subjects: Role of neural interlimb coupling. Clinical Neurophysiology vol. 127 2286-2293 (2016).

2. Collins, S. H., Bruce Wiggin, M. \& Sawicki, G. S. Reducing the energy cost of human walking using an unpowered exoskeleton. Nature 522, 212-215 (2015).

3. Combs, S. A., Dugan, E. L., Ozimek, E. N. \& Curtis, A. B. Bilateral coordination and gait symmetry after body-weight supported treadmill training for persons with chronic stroke. Clinical Biomechanics 28, 448-453 (2013).

4. Meijer, R. et al. Markedly impaired bilateral coordination of gait in post-stroke patients: Is this deficit distinct from asymmetry? A cohort study. Journal of NeuroEngineering and Rehabilitation 8, (2011).

5. Arya, K. N. \& Pandian, S. Interlimb neural coupling: Implications for poststroke hemiparesis. Annals of Physical and Rehabilitation Medicine vol. 57 696-713 (2014).

6. Roemmich, R. T. et al. Interlimb coordination is impaired during walking in persons with Parkinson's disease. Clinical Biomechanics 28, 93-97 (2013).

7. Krasovsky, T., Lamontagne, A., Feldman, A. G. \& Levin, M. F. Reduced gait stability in high-functioning poststroke individuals. J Neuro-physio/ 109, 77-88 (2013).

8. Reisman, D. S., Block, H. J. \& Bastian, A. J. Interlimb coordination during locomotion: What can be adapted and stored? Journal of Neurophysiology 94, 2403-2415 (2005).

9. Reisman, D. S., Wityk, R., Silver, K. \& Bastian, A. J. Locomotor adaptation on a split-belt treadmill can improve walking symmetry post-stroke. Brain 130, 1861-1872 (2007).

10. Plotnik, M., Giladi, N. \& Hausdorff, J. M. A new measure for quantifying the bilateral coordination of human gait: Effects of aging and Parkinson's disease. Experimental Brain Research 181, 561-570 (2007).

11. Plotnik, M., Giladi, N. \& Hausdorff, J. M. Bilateral coordination of walking and freezing of gait in Parkinson's disease. European Journal of Neuroscience 27, 1999-2006 (2008).

12. Awad, L. N., Kudzia, P., Revi, D. A., Ellis, T. D. \& Walsh, C. J. Walking Faster and Farther With a Soft Robotic Exosuit: Implications for Post-Stroke Gait Assistance and Rehabilitation. IEEE Open Journal of Engineering in Medicine and Biology 1, 108-115 (2020).

13. Galle, S., Malcolm, P., Collins, S. H. \& de Clercq, D. Reducing the metabolic cost of walking with an ankle exoskeleton: interaction between actuation timing and power. Journal of NeuroEngineering and Rehabilitation 14, (2017).

14. Kim, J. et al. Reducing the metabolic rate of walking and running with a versatile, portable exosuit Downloaded from. Science vol. 365 http://science.sciencemag.org/ (2019).

15. Takahashi, K. Z., Lewek, M. D. \& Sawicki, G. S. A neuromechanics-based powered ankle exoskeleton to assist walking post-stroke: A feasibility study. Journal of NeuroEngineering and Rehabilitation 12, (2015).

16. Awad, L. N. et al. A soft robotic exosuit improves walking in patients after stroke. Science Translational Medicine 9, (2017). 
17. Glaister, B. et al. Training for Patients Recovering from Neurological Injuries with Kickstart ${ }^{\circledR}$ : A Case Series. Physical Medicine and Rehabilitation-International 2, (2015).

18. Yao, J. et al. The Kickstart Walk Assist system for improving balance and walking function in stroke survivors: a feasibility study. Journal of Neuroengineering and Rehabilitation 18, 1-12 (2021).

19. Stergiou, N., Harbourne, R. T. \& Cavanaugh, J. T. Optimal Movement Variability: A New Theoretical Perspective for Neurologic Physical Therapy. Journal of Neurologic Physical Therapy vol. 120 https://journals.Iww.com/jnpt (2006).

20. Kelso, J. A. S. et al. A phase transition in human brain and behavior. Physics Letters A 169, 134-144 (1992).

21. Lamb, P. F. \& Stöckl, M. On the use of continuous relative phase: Review of current approaches and outline for a new standard. Clinical biomechanics (Bristol, Avon) 29, 484-493 (2014).

22. Park, K., Dankowicz, H. \& Hsiao-Wecksler, E. T. Characterization of spatiotemporally complex gait patterns using cross-correlation signatures. Gait and Posture 36, 120-126 (2012).

23. Loiret, l. et al. Are wearable insoles a validated tool for quantifying transfemoral amputee gait asymmetry? Prosthetics and orthotics international 43, 492-499 (2019).

24. Peters, B. T., Haddad, J. M., Heiderscheit, B. C., van Emmerik, R. E. A. \& Hamill, J. Limitations in the use and interpretation of continuous relative phase. Journal of Biomechanics vol. 36 (2003).

25. Coco, M. I. \& Dale, R. Cross-recurrence quantification analysis of categorical and continuous time series: An R package. Frontiers in Psychology 5, (2014).

26. McCamley, J., Denton, W., Lyden, E. \& Yentes, J. M. Measuring Coupling of Rhythmical Time Series Using Cross Sample Entropy and Cross Recurrence Quantification Analysis. Computational and Mathematical Methods in Medicine 2017, (2017).

27. Dutt-Mazumder, A., Rand, T. J., Mukherjee, M. \& Newell, K. M. Scaling oscillatory platform frequency reveals recurrence of intermittent postural attractor states. Scientific Reports 8, (2018).

28. Lake, D. E., Richman, J. S., Pamela Griffin, M. \& Randall Moorman, J. Sample entropy analysis of neonatal heart rate variability. Am J Physiol Regul Integr Comp Physio/ 283, 789-797 (2002).

29. Malcolm, P., Derave, W., Galle, S. \& de Clercq, D. A Simple Exoskeleton That Assists Plantarflexion Can Reduce the Metabolic Cost of Human Walking. PLOS ONE 8, (2013).

30. Henderson, G., Gordon, D. \& Vijayakumar, S. Identifying invariant gait metrics for exoskeleton assistance. https://ieeexplore.iee.org/document/8324469 (2017) doi:10.1109/ROBIO.2017.8324469.

31. Lee, S. H. et al. Gait performance and foot pressure distribution during wearable robot-assisted gait in elderly adults. Journal of NeuroEngineering and Rehabilitation 14, (2017).

32. Afsar, O., Tirnakli, U. \& Marwan, N. Recurrence Quantification Analysis at work: Quasi-periodicity based interpretation of gait force profiles for patients with Parkinson disease. Scientific Reports $\mathbf{8}$, (2018). 
33. Labini, F. S., Meli, A., Ivanenko, Y. P. \& Tufarelli, D. Recurrence quantification analysis of gait in normal and hypovestibular subjects. Gait and Posture 35, 48-55 (2012).

34. Mukherjee, M. \& Yentes, J. M. Movement variability: A perspective on success in sports, health, and life. Scandinavian Journal of Medicine and Science in Sports vol. 28 758-759 (2018).

35. Jordan, K., Challis, J. H. \& Newell, K. M. Long range correlations in the stride interval of running. Gait and Posture 24, 120-125 (2006).

36. Chien, J. H., Ambati, V. N. P., Huang, C. K. \& Mukherjee, M. Tactile stimuli affect long-range correlations of stride interval and stride length differently during walking. Experimental Brain Research 235, 1185-1193 (2017).

37. Hunt, N., McGrath, D. \& Stergiou, N. The influence of auditory-motor coupling on fractal dynamics in human gait. Scientific Reports 4, (2014).

38. Kaipust, J. P., Huisinga, J. M., Filipi, M. \& Stergiou, N. Gait Variability Measures Reveal Differences Between Multiple Sclerosis Patients and Healthy Controls. Motor Control vol. 16 (2012).

39. Lewek, M. D., Braun, C. H., Wutzke, C. \& Giuliani, C. The role of movement errors in modifying spatiotemporal gait asymmetry post stroke: a randomized controlled trial. Clinical Rehabilitation $\mathbf{3 2}$, 161-172 (2018).

40. Bastian, A. J. Understanding sensorimotor adaptation and learning for rehabilitation. Current Opinion in Neurology vol. 21 628-633 (2008).

41. Williams, A. J., Peterson, D. S. \& Earhart, G. M. Gait coordination in Parkinson disease: Effects of step length and cadence manipulations. Gait and Posture 38, 340-344 (2013).

42. Lee, S. J. \& Hidler, J. Biomechanics of overground vs. treadmill walking in healthy individuals. J App/ Physiol 104, 747-755 (2008).

43. Mukherjee, M. et al. Plantar tactile perturbations enhance transfer of split-belt locomotor adaptation. Experimental Brain Research 233, 3005-3012 (2015).

44. van den Bogert, A. J. Exotendons for assistance of human locomotion. http://www.biomedicalengineering-online.com/content/2/1/17 (2003).

45. Peng, H., Long, F. \& Ding, C. Feature Selection Based on Mutual Information: Criteria of MaxDependency, Max-Relevance, and Min-Redundancy. (2005).

46. Kennel, M. B., Brown, R. \& Abarbanel, H. D. I. Determining embedding dimension for phase-space reconstruction using a geometrical construction. Physical Review A 45, 3403-3411 (1992).

47. Shockley, K., Butwill, M., Zbilut, J. P. \& Webber, C. L. Cross Recurrence Quantification of Interpersonal Postural Activity. Physics Letters A 305, 59-69 (2002).

48. Zbilut, J. P. \& Webber, C. L. Embeddings and delays as derived from quantification of recurrence plots. (1992) doi:https://doi.org/10.1016/0375-9601(92)90426-M.

49. Wallot, S. Recurrence Quantification Analysis of Processes and Products of Discourse: A Tutorial in R. Discourse Processes 54, 382-405 (2017). 
50. Richman, J. S. \& Randall Moorman, J. Physiological time-series analysis using approximate entropy and sample entropy. Am J Physiol Heart Circ Physio/ 278, 2039-2049 (2000).

\section{Tables}

Table 1

Participant demographics. The two groups were not different in any of the demographics or measured walking speeds. NOTE: PWS = preferred walking speed.

\begin{tabular}{|c|c|c|c|c|}
\hline \multicolumn{4}{|c|}{ Independent t-test: $\mathrm{N}=18$ (EXO: $\mathrm{N}=9 /$ NO EXO: $\mathrm{N}=9$ ) } & \multirow{2}{*}{$\begin{array}{l}\text { T-test } \\
p \text {-value }\end{array}$} \\
\hline & Group & Mean & SD & \\
\hline \multirow[t]{2}{*}{ Age (years old) } & EXO & 23.2 & 3.0 & \multirow[t]{2}{*}{0.424} \\
\hline & NO EXO & 24.2 & 2.1 & \\
\hline \multirow[t]{2}{*}{ Height $(\mathrm{cm})$} & EXO & 177.6 & 5.9 & \multirow[t]{2}{*}{0.767} \\
\hline & NO EXO & 178.6 & 8.4 & \\
\hline \multirow[t]{2}{*}{ Mass $(\mathrm{kg})$} & EXO & 79.8 & 6.8 & \multirow[t]{2}{*}{0.974} \\
\hline & NO EXO & 79.9 & 14.0 & \\
\hline \multirow[t]{2}{*}{ PWS (m/s) } & EXO & 1.12 & 0.15 & \multirow[t]{2}{*}{0.734} \\
\hline & NO EXO & 1.10 & 0.10 & \\
\hline
\end{tabular}


Table 2

Mean of first 200 steps of linear measurements in each limb for the two groups. There was no significant difference between EXO and NO EXO.

\begin{tabular}{|c|c|c|c|c|}
\hline \multicolumn{5}{|l|}{ Independent t-test } \\
\hline & Group & Average & SD & $\mathrm{p}$-value \\
\hline \multirow[t]{2}{*}{ Step Length R (m) } & EXO & 0.59 & 0.06 & \multirow[t]{2}{*}{0.35} \\
\hline & NO EXO & 0.57 & 0.03 & \\
\hline \multirow[t]{2}{*}{ Step Length L (m) } & EXO & 0.59 & 0.06 & \multirow[t]{2}{*}{0.71} \\
\hline & NO EXO & 0.58 & 0.04 & \\
\hline \multirow[t]{2}{*}{ Step Time R (sec) } & EXO & 0.61 & 0.05 & \multirow[t]{2}{*}{0.07} \\
\hline & NO EXO & 0.58 & 0.03 & \\
\hline \multirow[t]{2}{*}{ Step Time L (sec) } & EXO & 0.57 & 0.04 & \multirow[t]{2}{*}{0.79} \\
\hline & NO EXO & 0.58 & 0.03 & \\
\hline \multirow[t]{2}{*}{ Step Width R (m) } & EXO & 0.17 & 0.02 & \multirow[t]{2}{*}{0.47} \\
\hline & NO EXO & 0.16 & 0.03 & \\
\hline \multirow[t]{2}{*}{ Step Width L (m) } & EXO & 0.17 & 0.02 & \multirow[t]{2}{*}{0.47} \\
\hline & NO EXO & 0.16 & 0.03 & \\
\hline
\end{tabular}

Table 3

Time delay and the EMB values for each participant in the EXO and the NO EXO group. These values were used to reconstruct state space figure and perform cRQA.

\begin{tabular}{|llllll|}
\hline Participant ID & Time Delay $(\tau)$ & EMB & Participant ID & Time Delay $(\tau)$ & EMB \\
\hline NOEXO04 & 3 & 5 & EXO01 & 3 & 5 \\
\hline NOEXO08 & 3 & 5 & EXO02 & 3 & 6 \\
\hline NOEXO09 & 3 & 5 & EXO03 & 3 & 5 \\
\hline NOEX010 & 3 & 5 & EXO04 & 3 & 5 \\
\hline NOEX013 & 3 & 4 & EXO10 & 4 & 5 \\
\hline NOEX015 & 3 & 5 & EX011 & 3 & 5 \\
\hline NOEX016 & 3 & 5 & EXO13 & 3 & 5 \\
\hline NOEX021 & 3 & 5 & EXO14 & 4 & 5 \\
\hline NOEXO32 & 3 & 5 & EXO17 & 3 & 6 \\
\hline
\end{tabular}




\section{Figures}

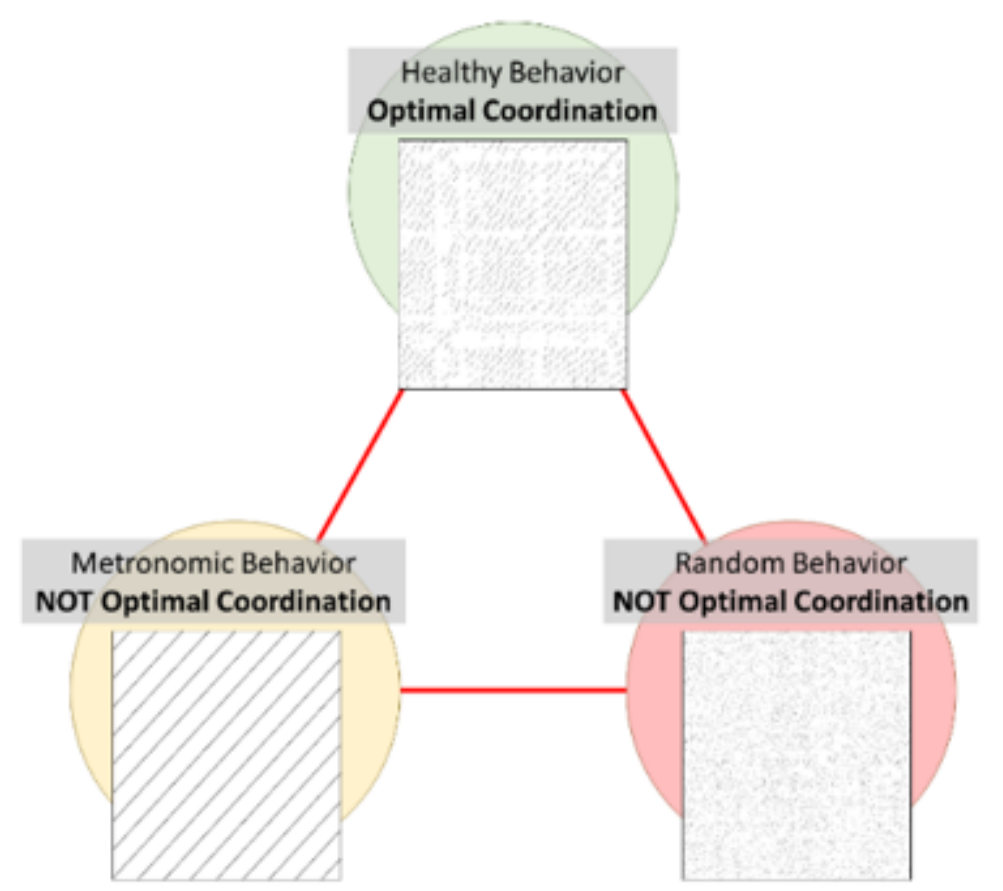

\section{Figure 1}

shows the model of inter-limb coordination. In this model, healthy inter-limb coordination sits at a sweetspot between patterns that exhibit either metronomic qualities or random-like qualities. Healthy individuals demonstrate optimal coordination (top) where the cross recurrence plot shows an intermediate level of diagonal lines (representative recurrence plot from the control group). Diagonal lines indicate the length of time that the right and left limbs are coordinated. Longer or shorter diagonal lines indicate a non-optimal coordinated behavior (bottom left and right). Bottom left shows the recurrence plot with two simulated sinusoidal time series and long diagonal lines, as would be seen in perfectly coordinated gait. Bottom right shows the opposite, an uncoordinated pattern of two simulated random time series with negligible diagonal lines. 

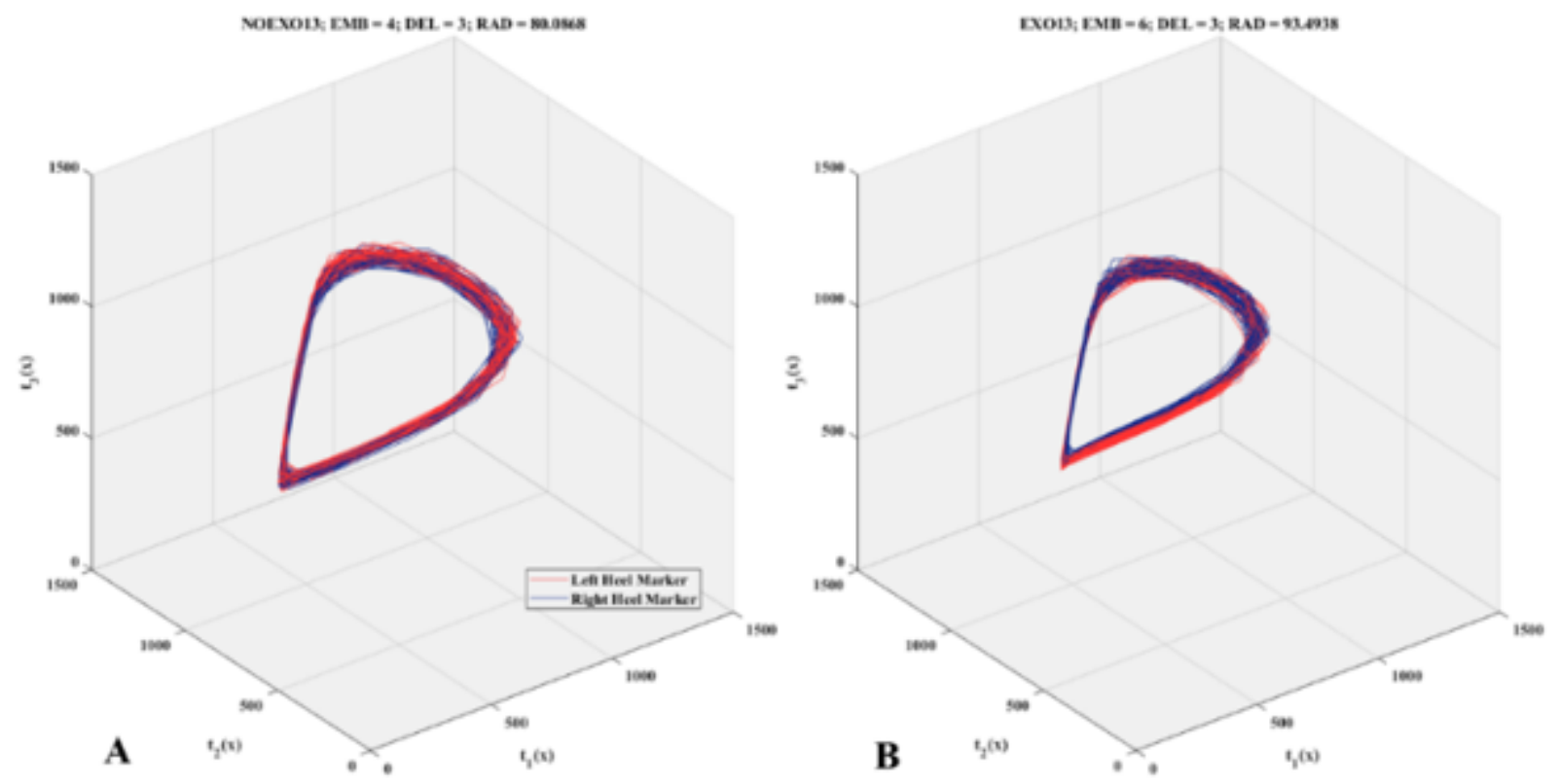

Figure 2

A and $B$ shows the state space reconstruction figure of one participant in each group. There are reconstruction figures for left heel, right heel, and both of them combined. Figure $3 \mathrm{~A}$ shows the reconstruction figure of a person walking without an exoskeleton, and Figure 3B shows the figure of a person with the exoskeleton.
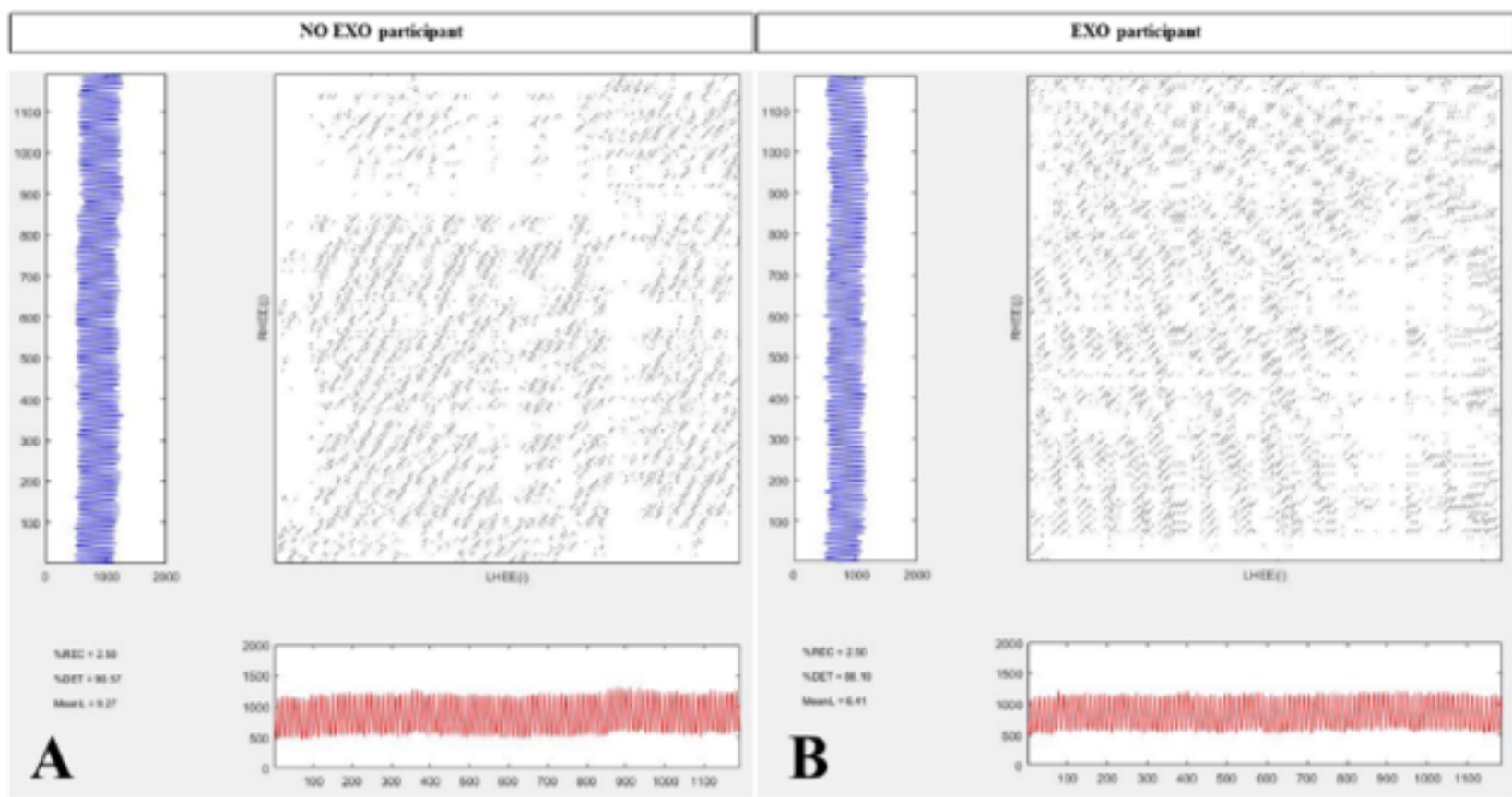

Figure 3 
A and $B$ shows the recurrence plot of one participant in each group. These are the same participants as in figure 3. Figure $5 \mathrm{~A}$ shows the recurrence plot of a person walking without an exoskeleton. Figure $5 \mathrm{~B}$ shows the recurrence plot of a person with the exoskeleton.
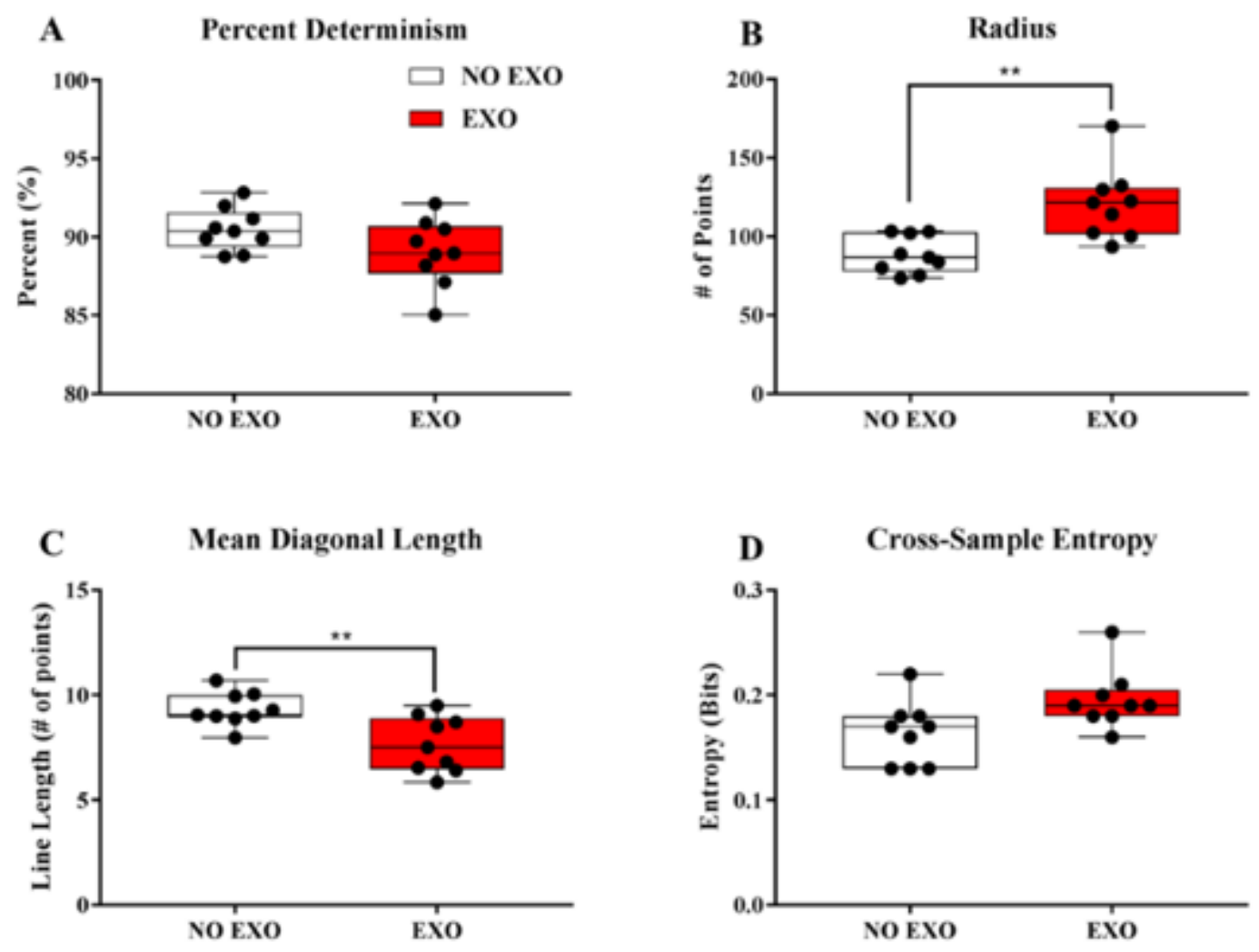

Figure 4

A-D shows box and whisker plots. A-C shows the main CRQA variables that were analyzed. Figure 6D shows the entropy value from cSE. Each dot represents a value of each participant, and error bar shows the standard deviation. Significant differences between the EXO and the NO EXO group are shown. ${ }^{*} p<0.05,{ }^{*} \mathrm{p}<0.01$, and ${ }^{* * *} \mathrm{p}<0.001$. 


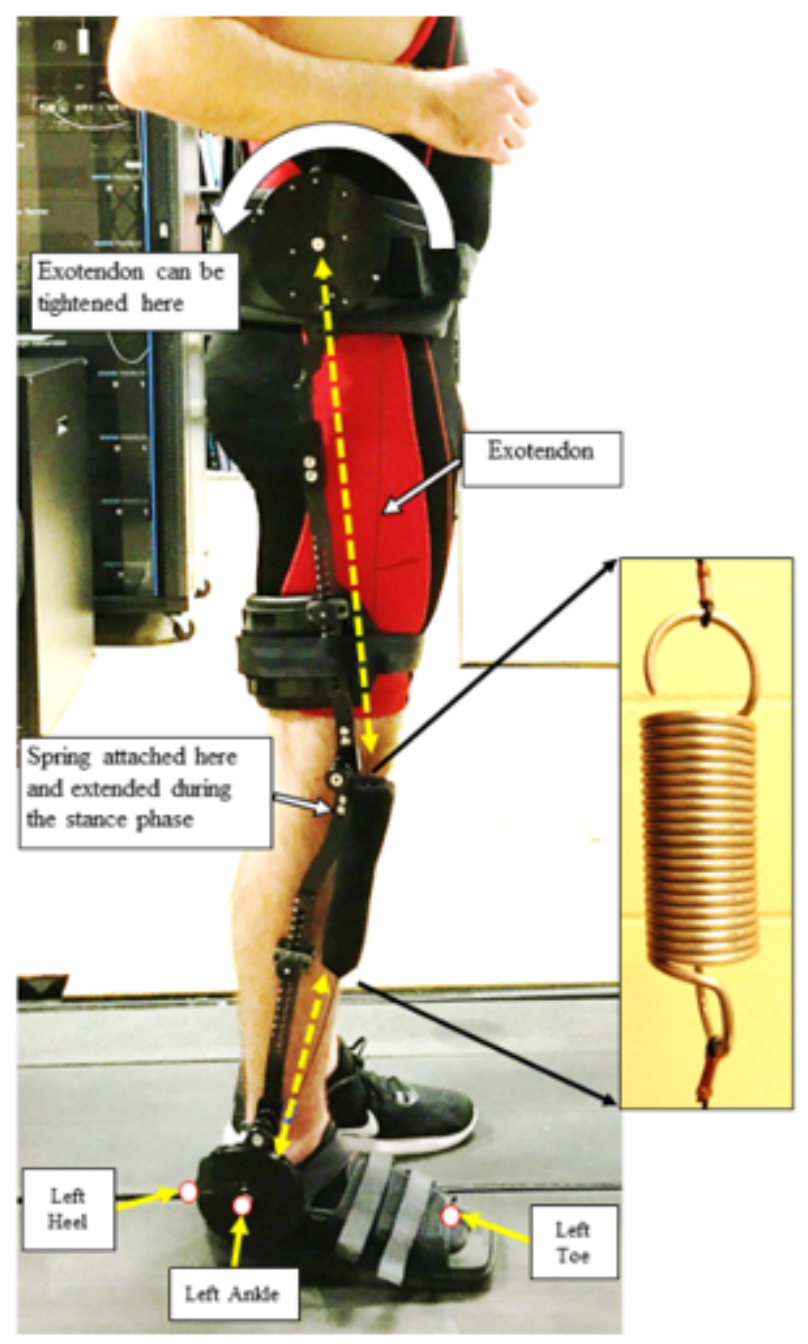

Figure 5

shows a device set up. Figure 2A shows a participant with the passive exoskeleton attached to their right dominant leg. The spring is attached to a cable called the Exotendon, which can be tightened by the disk on the waist. While a person is in the stance phase of gait, the spring is extended, storing energy. When a person propels forward, the spring provides assistance by returning the energy. The figure also shows the positions of the retro-reflective feet markers that were used in the study. 


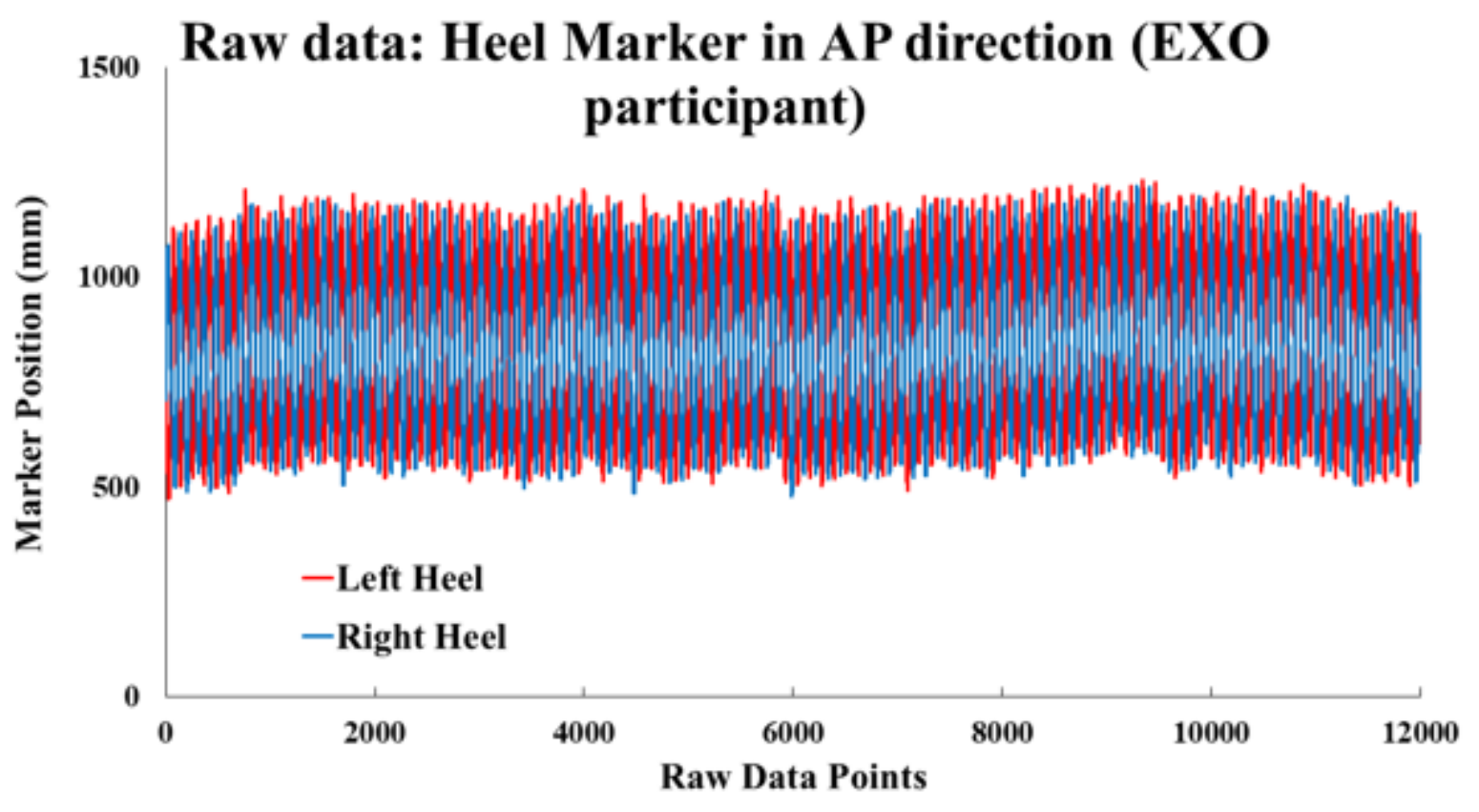

Figure 6

shows a raw position data in $\mathrm{mm}$ of a representative participant's right and left heel marker in the AP direction. 